\title{
EVEN CONTINUTTY AND THE BANACH CONTRACTION PRINCIPLE
}

\section{J. L. SOLOMON AND LUDVIK JANOS}

\begin{abstract}
In 1967, Philip R. Meyers established a nice converse to the Banach Contraction Mapping Theorem. We provide a counterexample to one of his corollaries and show that if $X$ is a metrizable topological space, $f$ a continuous self-map on $X$ such that: (a) $f$ has a fixed point $p$ which has a compact neighborhood; (b) $f^{n}(x) \rightarrow p$ as $n \rightarrow \infty$ for each $x$ in $X$, then the following are equivalent: (1) $f$ is a contraction relative to a suitable metric on $X$; (2) the sequence of iterates $\left\{f^{n}\right\}_{n=1}^{\infty}$ is evenly continuous.
\end{abstract}

Throughout this paper, $X$ will denote a metrizable topological space and $f$ a continuous self-map on $X$. For later reference, we list the following conditions:

(A) there exists an element $p$ in $X$ such that $f(p)=p$;

(B) for each $x$ in $X, f^{n}(x) \rightarrow p$ as $n \rightarrow \infty$; and,

(C) there exists an open neighborhood $U$ of $p$ such that for each open neighborhood $V$ of $p$ there exists a positive integer $N=N(V)$ such that $f^{n}(U) \subset V$ for each $n \geqslant N$.

In 1967, Philip R. Meyers [2] established the following result.

THEOREM 1. If $f$ satisfies (A), (B), and (C) then for each $\lambda \in(0,1)$, there exists a metric $d_{\lambda}$ on $X$, complete if $X$ is topologically complete, such that $f$ is a $d_{\lambda}$-contraction with contraction constant $\lambda$.

Meyers includes the following corollary.

COROLlARY 1. If $p$ has a compact neighborhood then conditions (A) and (B) are sufficient for Theorem 1.

The proof of Corollary 1 is in error and, as the following example shows, the corollary is, in fact, false. (The error was recognized and corrected by deletion in the correction list [2].)

EXAMPLE 1. Let $X$ be the unit circle in the complex plane and let $f: X \rightarrow X$ be defined by $f\left(e^{2 \pi i \alpha}\right)=\exp \left(2 \pi i \alpha^{2}\right)$ for $\alpha \in[0,1]$. It is evident that $f$ satisfies (A) and (B) with 1 the unique fixed point. Since $f$ is a surjection we have $X=f(X)$ implying that for any metric on $X$ the diameter of $X$ coincides with that of $f(X)$. Due to compactness of $X$ this diameter is finite, which in turn implies that $f$ cannot be a contraction relative to any equivalent metric for $X$.

Received by the editors March 30, 1977 and, in revised form, July 28, 1977.

AMS (MOS) subject classifications (1970). Primary 54E40; Secondary 54H25.

Key words and phrases. Banach contraction principle, evenly continuous family, fixed point. 
By careful examination of the error committed in the proof of Corollary 1, one seeks for some additional assumptions to make this statement true. The purpose of this note is to show that the even continuity of the family $\left\{f^{n}\right.$ : $n \geqslant 1$ \} of iterates of $f$ is one such possible assumption. For completeness we give here the general definition of even continuity (see Kelley [1]).

Definition 1 . Let $F$ be a family of functions, each on a topological space $X$ to a topological space $Y$. The family $F$ is evenly continuous if and only if for each $x$ in $X$, each $y$ in $Y$, and each open neighborhood $U$ of $y$ there is an open neighborhood $V$ of $x$ and an open neighborhood $W$ of $y$ such that $f(V) \subset U$ whenever $f(x) \in W, f \in F$.

REMARK. It is necessary to work with the topological concept of even continuity rather than with the metric-dependent equicontinuity, since the formulation of our question is purely topological.

We shall need the following lemma, which is a special case of a problem in Kelley [1, p. 235].

LEMMA 1. Let $f: X \rightarrow X$ be such that the family of iterates $\left\{f^{n}: n \geqslant 1\right\}$ of $f$ is evenly continuous. Assume that a sequence $\left\{x_{n}\right\} \subset X$ converges to $x$ in $X$ and $\left\{f^{k_{n}}\right\}$ is a subsequence of $\left\{f^{n}\right\}$ with $f^{k_{n}}(x)$ converging to some point $y$ in $X$. Then $f^{k_{n}}\left(x_{n}\right) \rightarrow y$.

We are now ready to state our theorem.

THEOREM 2. Let $X$ be a metrizable topological space and $f$ a continuous self-map such that: (i) $f$ has a fixed point, $p$, which has an open neighborhood with compact closure; (ii) for every $x$ in $X$, the sequence $\left\{f^{n}(x)\right\}$ converges to $p$. Then the following statements are equivalent.

(1) For each $\lambda \in(0,1)$, there exists a metric $d_{\lambda}$ on $X$, complete if $X$ is topologically complete, such that $f$ is a $d_{\lambda}$-contraction with contraction constant $\lambda$.

(2) The sequence of iterates of $f$ is evenly continuous.

Proof. It is straightforward to show that (1) implies (2). Thus all we need is to show that (2) implies (1). Let $U$ be an open neighborhood of $p$ such that the closure of $U, \operatorname{cl}(U)$, is compact. Consider any open neighborhood $V$ of $p$. For $x \in \operatorname{cl}(U)$, let $N(x)$ denote the least positive integer such that $n \geqslant N(x)$ implies $f^{n}(x) \in V$. Now, define $N=\sup \{N(x): x \in \operatorname{cl}(U)\}$. Let us assume that $N$ is infinite. Then there exists a sequence $\left\{x_{n}\right\}$ in $\operatorname{cl}(U)$ such that $N\left(x_{n}\right)$ is monotone increasing. Due to compactness of $\operatorname{cl}(U)$, we may assume that $x_{n} \rightarrow x$ in $\operatorname{cl}(U)$. Define $k_{n}=N\left(x_{n}\right)-1$. We see that $k_{n} \rightarrow \infty$ and $f^{k_{n}}\left(x_{n}\right) \notin$ $V$ for any $n$. From (ii) we have that $f^{k_{n}}(x) \rightarrow p$. Since $x_{n} \rightarrow x$ and $\left\{f^{n}\right\}$ is evenly continuous, it follows from Lemma 1 that $f^{k_{n}}\left(x_{n}\right) \rightarrow p$ as $n \rightarrow \infty$, contradicting the fact that $f^{k_{n}}\left(x_{n}\right) \notin V$ for any $n$. Thus $N$ is finite and $f^{n}(U) \subset V$ for each $n \geqslant N$. Hence conditions (A), (B), and (C) are satisfied and by Theorem 1, we have that (2) implies (1) and our assertion follows. 


\section{REFERENCES}

1. J. L. Kelley, General topology, Van Nostrand, New York, 1955.

2. P. R. Moyers, A conoerse to Banach's contraction theorem, J. Res. Nat. Bur. Standarde Sect. B 71B (1967), pp. 73-76.

Departnent of Mathematics, Mississippi State University, Mississippi State, Mississippi 39762 